\title{
Wind Power Prediction Based on LS-SVM Model with Error Correction
}

\author{
Yagang ZHANG ${ }^{1,2}$, Penghui WANG ${ }^{1}$, Tao NI ${ }^{1}$, Penglai $\mathrm{CHENG}^{1}$, Shuang LEI ${ }^{1}$ \\ ${ }^{1}$ State Key Laboratory of Alternate Electrical Power System with Renewable Energy Sources, North \\ China Electric Power University, Beijing, 102206, China \\ ${ }^{2}$ Interdisciplinary Mathematics Institute, University of South Carolina, \\ Columbia, SC 29208, United States \\ yagangzhang@ncepu.edu.cn
}

\begin{abstract}
As conventional energy sources are nonrenewable, the world's major countries are investing heavily in renewable energy research. Wind power represents the development trend of future energy, but the intermittent and volatility of wind energy are the main reasons that leads to the poor accuracy of wind power prediction. However, by analyzing the error level at different time points, it can be found that the errors of adjacent time are often approximately the same, the least square support vector machine (LS-SVM) model with error correction is used to predict the wind power in this paper. According to the simulation of wind power data of two wind farms, the proposed method can effectively improve the prediction accuracy of wind power, and the error distribution is concentrated almost without deviation. The improved method proposed in this paper takes into account the error correction process of the model, which improved the prediction accuracy of the traditional model (RBF, Elman, LS SVM). Compared with the single LS-SVM prediction model in this paper, the mean absolute error of the proposed method had decreased by 52 percent. The research work in this paper will be helpful to the reasonable arrangement of dispatching operation plan, the normal operation of the wind farm and the large-scale development as well as fully utilization of renewable energy resources.
\end{abstract}

Index Terms - computer errors, error correction, support vector machines, power engineering computing, wind energy generation.

\section{INTRODUCTION}

In the 1970s, the emergence of energy shortage in the world powers has made people aware of the need to find and develop new energy sources in order to survive. Many countries begun to set energy policy and actively engaged in the adjustment of energy structure, greenhouse gas emissions reduce, the strengthening of environmental pollution mitigation and energy security and thus the development of renewable energy using has been highly valued. In these new energies, wind energy is considered to be the most mature technology, commercialization of it has been basically achieved and it is the most potential renewable energy technology. At present, wind power is one of the ways to use wind energy in large scale. According to data released by the Global Wind Energy Association (GWEC), from 2006 to the end of 2015, the global wind

This research was supported partly by the National Key Basic Research Project (973 Program) of China (2012CB215200), the National Natural Science Foundation of China (51637005), the Fundamental Research Funds for the Central Universities (2017MS33) and the Natural Science Foundation of Hebei Province (G2016502009). power installed capacity had increased from $74 \mathrm{GW}$ to $432.9 \mathrm{GW}$, about 5 times. At the same time the International Energy Agency (IEA) pointed out that in 2015, under the premise that the growth rate of global economy is higher than the $3 \%$, global carbon emissions related to energy amount about 322 million tons, essentially flat with 322 million tons in 2014. This is mainly due to the use of green energy in more countries.

Due to the advantages of wind energy and the negative impact of fossil consumption on climate change, wind power market has good prospects for development. With the development of wind power, power system is facing complex changes in all aspects, while the accurate daily power scheduling is the key to change the original energy structure and implementation of the new energy plan. High precision estimation of future power output of wind farm can reasonably arrange power dispatching plan and reduce operation cost of power system. Therefore, accurate prediction of wind power is the key to optimize the grid scheduling, reduce the cost of economic operation and ensure normal operation of wind farm.

Many scholars have put forward a lot of methods to improve the wind power prediction. At present, the prediction model of wind power is roughly divided into four categories: physical model, statistical model, artificial intelligence technology and hybrid model. Physical model [1] is based on the improvement of the resolution of numerical weather prediction (NWP), combined with the process of geographical factors. Numerical weather forecast is difficult to obtain, it needs to be supported by the abundant data in the field of statistics or meteorology. Statistical model [2-4] is a mapping relationship between the input of the system and the power of wind power generation. The commonly used methods mainly include Autoregressive moving average model (ARMA), generalized ARCH model (GARCH), grey prediction, empirical mode decomposition, Kalman filter model, etc. An autoregressive moving average model (ARMA-GARCH) was proposed to predict wind speed [5]. The results show that the proposed method can effectively describe the characteristics of wind energy. Based on the grey model, an effective and feasible method of grey correlation weighted combination prediction model was proposed in [6]. Commonly used artificial intelligence technologies [7-9] are the radial basis function neural network (RBF), the wavelet neural network (WNN), support vector machine (SVM), the fuzzy forecast method and so on. The uncertainty of wind speed and its inherent volatility 
make the prediction accuracy of the single model not ideal and the processing and use of data of each model are not the same. Therefore, each method has some limitations. In order to get higher prediction accuracy, researchers put forward some models based on the combination of two or more than two kinds of prediction methods and had got new research results. Self-Organizing Maps (SOM) and Multi-layer perceptron (MLP) were combined into a computational model to get a reliable prediction result in [10]. Combined with the support vector machine model of the state space and the unscented Kalman filter of the dynamic state estimation, a new hybrid model is proposed [11]. The model shows good robustness. But in order to meet the accuracy requirement of the wind farm power prediction system, a higher accuracy prediction model is needed.

In the prediction of wind power, we have carried out a lot of research work. In paper [12], the dynamic characteristic of the atmospheric system was introduced into the research and a new wind prediction method based on wavelet neural network was adopted, which significantly improved the prediction of wind power. Based on grey generating and an accumulated generating model, a new wind disturbance model to improve the wind forecasting precision of BP network methods is proposed [13]. In paper [14], the Lorenz system is the theoretical basis of the atmospheric disturbance, and a variety of artificial neural network models are used to verify the short-term wind power prediction and the feasibility analysis of the Lorenz system.

In most cases, due to the characteristics of self-adaptation and self-learning, intelligent algorithm [15-17] is more able to adapt to the nonlinear change of wind, such as neural network (ANN) and support vector machine (SVM), etc. But these methods are almost all based on the prediction model of the optimization numerical algorithm, ignoring the actual movement of the atmospheric system. Therefore, based on the traditional SVM method, considering the characteristics of wind energy forecasting, the least square support vector machine (LS-SVM) model based on the error correction is proposed in this paper. Compared with the traditional prediction methods, the prediction effect of the method proposed above is very significant.

The structure of this paper is as follows: the second section mainly introduces the least square support vector machine algorithm and error correction principle; The third section introduces the modeling data and evaluation index of two wind farms; the fourth section describes the power of the prediction results and error analysis of the simulation of two wind farm power data at home and abroad and the fifth section summarizes the full text and makes a prospect.

\section{Wind Power Prediction Model of LS-SVM BASED ERROR CORRECTION}

SVM is a new kind of machine learning method based on statistical theory. By using nonlinear kernel function, SVM maps the input sample space to high dimension linear feature space, making the classification and regression of processing highly nonlinear possible. SVM had overcome the shortcomings of long training time, poor generalization ability and easily trapping into local minimum points found in artificial neural network. SVM improves the ability of learning and generalization of the model.
Combined with the related research of SVM [18], the researchers put forward the LS-SVM, LS-SVM is an upgrade for the SVM, which changes the traditional SVM inequality constraints into equality constraints and taking the error squared and the loss function as the training set experience loss. In this way, solving the two programming is transformed into solving linear equations, which improves the speed and convergence of the solution [19].

Supposing the training data set as $\left\{\left(x_{i}, y_{i}\right) \mid i=1,2, \ldots, l\right\}$, where $l$ represents the total number of samples, $x_{i} \in R^{n}$ represents input variables, $y_{i} \in R$ represents output variables, $n$ is the dimension of $x_{i}$. According to the theory of support vector machine, mapping the samples from the original space $R^{n}$ to the feature space $\varphi\left(x_{i}\right)$ by a nonlinear mapping $\varphi(\bullet)[20]$, the optimal decision function is constructed in high dimensional space as:

$$
y(x)=\omega^{T} \varphi(x)+b
$$

$\varphi(x)$ is the nonlinear function; $\omega$ is the weight; $b$ is the constant, $\omega$ and $b$ are uncertain parameters.

By using the structural risk minimization principle, $\omega$, $b$ can be found. The objective function of the LS-SVM optimization problem [21-22] is defined as:

$$
\min _{\omega, \xi} J(\omega, \xi)=\frac{1}{2} \omega^{T} \omega+\frac{1}{2} r \sum_{i=1}^{l} \xi_{i}^{2}
$$

Subject to:

$$
y_{i}=\varphi\left(x_{i}\right) \omega+b+\xi_{i} \quad(i=1,2, \ldots, l)
$$

$\xi_{i}$ is a relaxation factor, which represents the error between actual output and predictive output; $r$ is the penalty parameter, which control the degree of punishment beyond the error of the sample. The Lagrange function is constructed as:

$$
\begin{array}{r}
L(\omega, b, \xi, a)=\frac{1}{2} \omega^{T} \omega+\frac{1}{2} r \sum_{i=1}^{l} \xi_{i}^{2}- \\
\sum_{i=1}^{l} a_{i}\left(\varphi\left(x_{i}\right) \omega+b+\xi-y_{i}\right)
\end{array}
$$

$a_{i}$ is the Lagrange multiplier.

According to KTT:

$$
\left\{\begin{array}{l}
\frac{\partial L}{\partial \omega}=0 \Rightarrow \omega=\sum_{i=1}^{l} a_{i} \varphi\left(x_{i}\right) \\
\frac{\partial L}{\partial b}=0 \Rightarrow \sum_{i=1}^{l} a_{i}=0 \\
\frac{\partial L}{\partial \xi}=0 \Rightarrow a_{i}=r \xi_{i} \\
\frac{\partial L}{\partial a}=0 \Rightarrow \omega^{T} \varphi\left(x_{i}\right)+b+\xi_{i}-y_{i}=0
\end{array}\right.
$$

$\omega, \xi$ are eliminated, given by:

$$
\left[\begin{array}{ll}
0 & 1^{T} \\
1 & K+r^{-1} E
\end{array}\right]\left[\begin{array}{l}
b \\
a
\end{array}\right]=\left[\begin{array}{l}
0 \\
y
\end{array}\right]
$$


Where $1=[1,1, \ldots, 1]^{T}$ is a $l$ dimension vectors. $E$ is unit array of $l$ dimension. $a$ and $b$ represent the parameters of the LS-SVM model, $a=\left[a_{1}, a_{2}, \ldots, a_{l}\right], b$ is a constant; $K$ is an $l \times l$ kernel function symmetric matrix, and $K_{i j}=\varphi\left(x_{i}\right)^{T} \varphi\left(x_{j}\right)=K\left(x_{i}, x_{j}\right)$, where $K\left(x_{i}, x_{j}\right)$ is the kernel function that satisfy the Mercer condition.

The least square method is used to obtain the formula (6) and the corresponding parameters are given as:

$$
\left\{\begin{array}{l}
b=\frac{1^{T}\left(K+\frac{E}{r}\right)^{-1}}{1^{T}\left(K+\frac{E}{r}\right)^{-1} 1} \\
a=\left(K+\frac{E}{r}\right)^{-1}(y-b 1)
\end{array}\right.
$$

From the above formula, we can get the regression equation of the LS-SVM model:

$$
f\left(x_{j}\right)=\omega \varphi(x)+b=\sum_{i=1}^{l} a_{i} K\left(x_{i}, x_{j}\right)+b
$$

$K\left(x_{i}, x_{j}\right)$ is called a kernel function, $a$ and $b$ are obtained from formula (7).

At present, the research scholars pay less attention to the error of wind prediction. However, by analyzing the error level at different time points, it can be found that the error of adjacent time is often approximately the same [23]:

$$
\tilde{E}_{t+1}=E_{t}
$$

$E_{t}$ denotes the error of the measured value $P_{t}$ and predictive value $\tilde{P}_{t}$ at time $t$ :

$$
E_{t}=P_{t}-\tilde{P}_{t}
$$

The power predictive value $\hat{P}_{t+1}$ at the next time $t+1$ after the correction is

$$
\hat{P}_{t+1}=\tilde{P}_{t+1}+\tilde{E}_{t+1}=\tilde{P}_{t+1}+E_{t}
$$

$\tilde{P}_{t+1}$ denotes the predictive value at time $t+1$, $\tilde{E}_{t+1}$ denotes the measured value at time $t+1, E_{t}$ denotes the error at time $t$.

\section{DATA DESCRIPTION AND ERROR INDEX OF THE SIMULATION}

In order to verify the validity of the LS-SVM wind prediction model with error correction, the power data of two wind farms at home and abroad were chosen to carry out experiments. 1107 groups of data from Spanish wind farm are as shown in Figure 1(a), the first 1007 sets of the data constitute the training set and the rest are the prediction set; As shown in Figure 1(b), there are 506 sets of power data from wind farm in China, where the first 456 sets of data are the training set and the rest are the prediction set. The power data of the two wind farms in Figure 1 were measured at intervals of $10 \mathrm{~min}$, the horizontal coordinate represents the predicted time point, and the longitudinal coordinate indicates the power value. The scatter of wind speed and wind power of the wind farm is plotted in Figure 2 , it can be seen that the power and speed distribution of the two wind farms are random and show the obvious nonlinear characteristics.

In order to make a more accurate evaluation of the LSSVM model with error correction, mean absolute error (MAE), mean square error (MSE) and mean absolute percent error (MAPE) are selected to evaluate the prediction results quantitatively in this paper. The specific formulas are as follows:

$$
\begin{aligned}
& M A E=\frac{1}{n} \sum_{t=1}^{n}|P(t)-\hat{P}(t)| \\
& M S E=\frac{1}{n} \sum_{t=1}^{n}(P(t)-\hat{P}(t))^{2} \\
& M A P E=\frac{1}{n} \sum_{t=1}^{n}\left|\frac{P(t)-\hat{P}(t)}{P(t)}\right| \times 100
\end{aligned}
$$

$P_{t}$ and $\hat{P}(t)$ respectively denote the actual value and predictive value of the moment power.

The LS-SVM modeling with error correction process is as follows:

First, this paper used MATLAB software to write LSSVM power prediction model, and selected radial basis function as the kernel function of the LS-SVM model, as shown in formula (13). And the grid search method is used to determine the parameters in the program, the optimal parameters were shown in table 1 . After the training of LSSVM model, the initial value $\tilde{P}_{t}$ of power prediction is obtained.

$$
K\left(x_{i}, x_{j}\right)=\exp \left(-\frac{\left\|x_{i}-y_{j}\right\|}{2 \sigma^{2}}\right)
$$

TABLE I. THE PARAMETERS SET OF LS-SVM MODEL

\begin{tabular}{|c|c|c|}
\hline \multirow{2}{*}{ Wind farm } & \multicolumn{2}{|c|}{ parameters } \\
\cline { 2 - 3 } & $\sigma$ & $r$ \\
\hline Wind farm in Spanish & 0.005 & 9 \\
\hline Wind farm in China & 0.0101 & 13 \\
\hline
\end{tabular}

Second, the error correction model is used to correct the initial power prediction value $\tilde{P}_{t}$ at time $t$, and the correction power $\hat{P}_{t}$ is obtained by:

$$
\hat{P}_{t}=\tilde{P}_{t}+E_{t-1}=\tilde{P}_{t}+\left(P_{t-1}-\tilde{P}_{t-1}\right)
$$

Where $P_{t-1}$ denotes the measure value at time $t-1$; $\tilde{P}_{t-1}$ denotes the initial prediction value at time $t-1$.

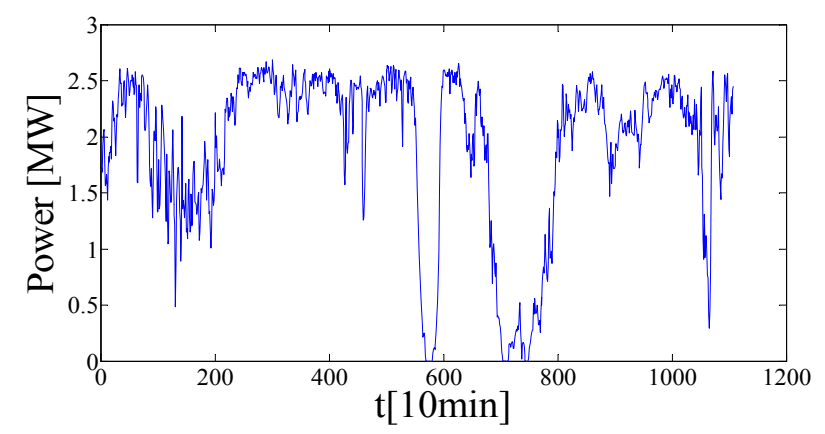

(a) 


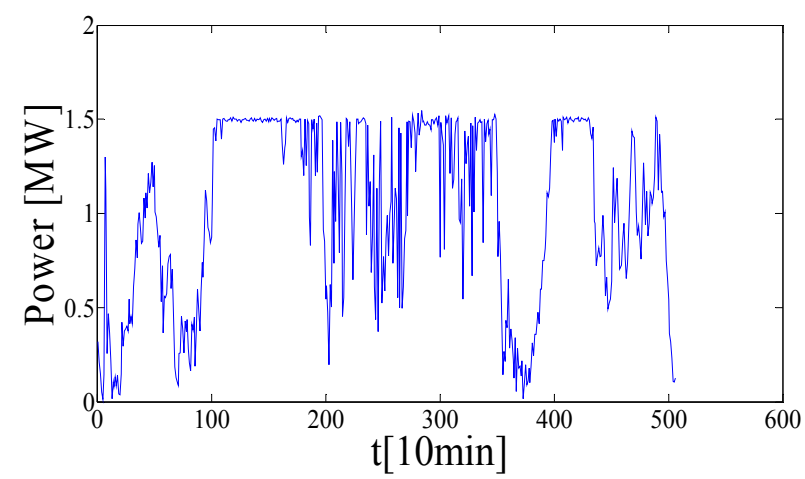

(b)

Figure 1. Power distribution of wind farm. (a) Wind farm in Spain, (b) Wind farm in China.

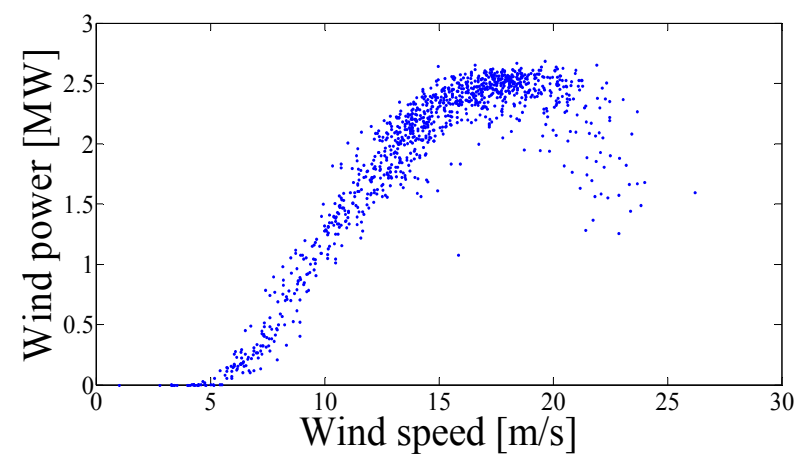

(a)

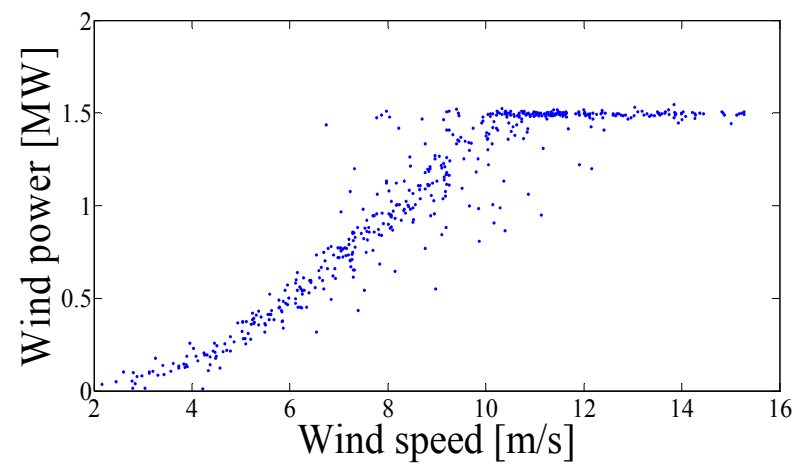

(b)

Figure 2. Scatter of wind power and wind speed. (a) Wind farm in Spain, (b) Wind farm in China.

\section{PRediction Results ANd ERror AnAlysis OF LS- SVM WITH ERROR CORRECTION}

Based on the description of the prediction algorithm in section II, and the modeling process in section III of, the LSSVM power prediction model and the error correction power prediction model of LS-SVM for two farms were established, and RBF neural network model as well as Elman neural network prediction model were established as the reference model. Figure 3 shows the power prediction curve for four models of two wind farms. The red curve with $\Delta$ represents the initial forecast results of LS-SVM model; green curve with o represents the prediction results of LS-SVM model with error correction and the black line represents the actual power. Due to the weak relationship between wind power output and wind speed, RBF, Elman and LS-SVM model have not shown good performance, especially in the place where the power series is more volatile. The power distribution of the wind farm in Spain is relatively stable, with only little fluctuations, so the forecast effect is more obvious than that of the wind farm in China.
From the graph, it also can be seen that although the overall trend of the traditional prediction models (RBF, Elman, LSSVM) are basically consistent with the original data, the green curve is more close to the original power curve, maintaining the overall trend of the raw data, which shows that the LS-SVM model is more close to the actual power curve after the error correction and can reflect the true power better.

As shown in Figure 3, the randomness and volatility of wind make the LS-SVM model not show a good predictive performance, and the prediction results of the modified LSSVM model are satisfactory. The prediction error of the LSSVM model both before and after the correction of the wind farm in Spain is shown in Figure 4(a), and the error distribution is shown in figure 4(b1) and 4(b2). The blue solid line shows the prediction error of LS-SVM before the correction and the red solid line shows the prediction after the error correction. Although the most errors of the blue line are in $[-1.5 \mathrm{MW}, 1 \mathrm{MW}]$, the error distribution in Figure 4(a) is larger and positively biased, which reveals the poor forecasting performance. While the red curve in Figure 4(a) in the vicinity of 0 fluctuation and the errors are basically in [-0.5MW, 0.5MW]. The error distribution in Figure 4(b2) is concentrated and almost unbiased, indicating the significant effect of error correction in LS-SVM model.

Also, Figure 5 shows the data error and distribution of the LS-SVM model before and after the error correction for wind farm in China. As shown in figure 5(a), the blue line significantly deviated from the horizontal line, while the forecast error after error correction is more evenly distributed on both sides of the horizontal line; Error distribution range in Figure 5(b1) is large and in the range of [-0.6MW, 0.4MW], while Figure 5(b2) shows that most of prediction errors lie in the interval of $[-0.4 \mathrm{MW}, 0.3 \mathrm{MW}]$ and the distribution is basically concentrated, which also can prove that the LS-SVM prediction model with error correction is effective.

(a)
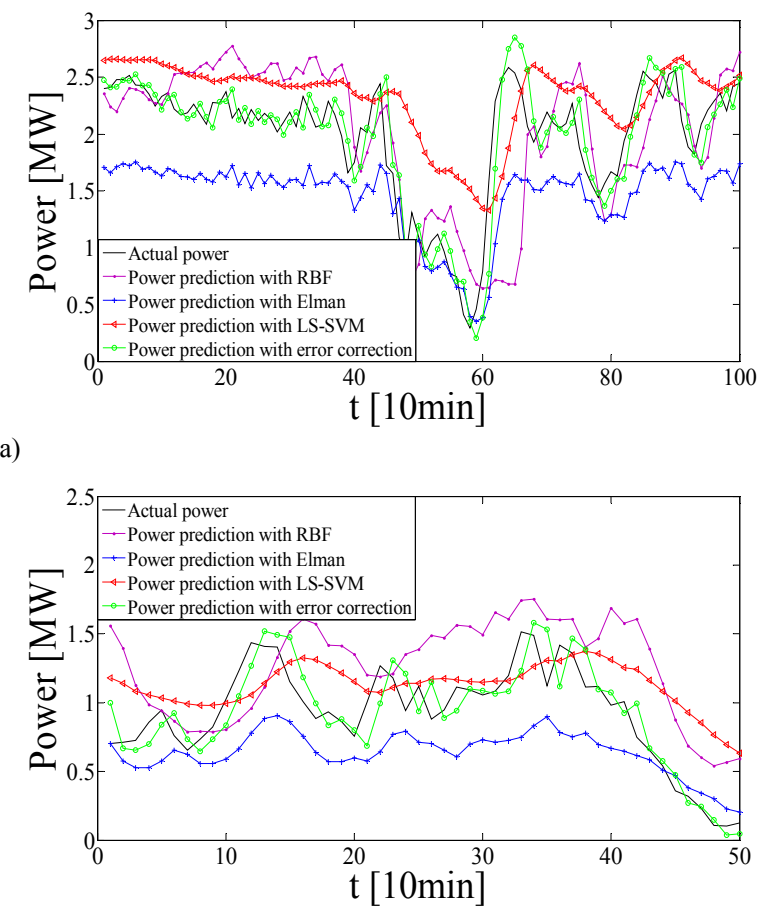

(b)

Figure 3. Power prediction of LS-SVM models with error correction and not. (a) Wind farm in Spain, (b) Wind farm in China. 


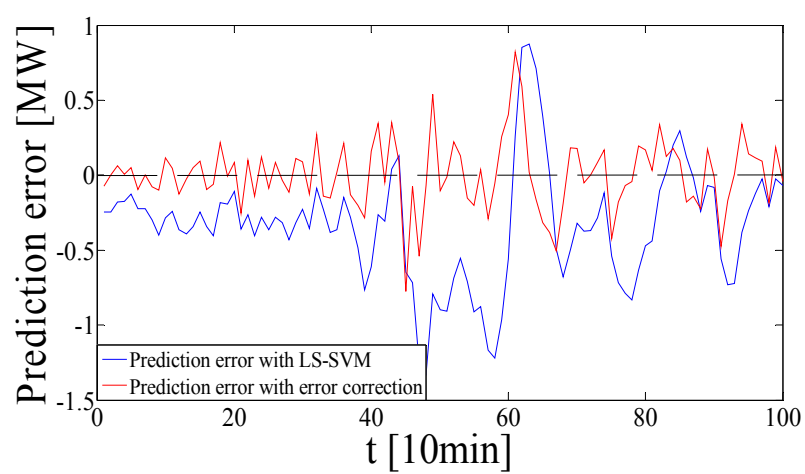

(a)

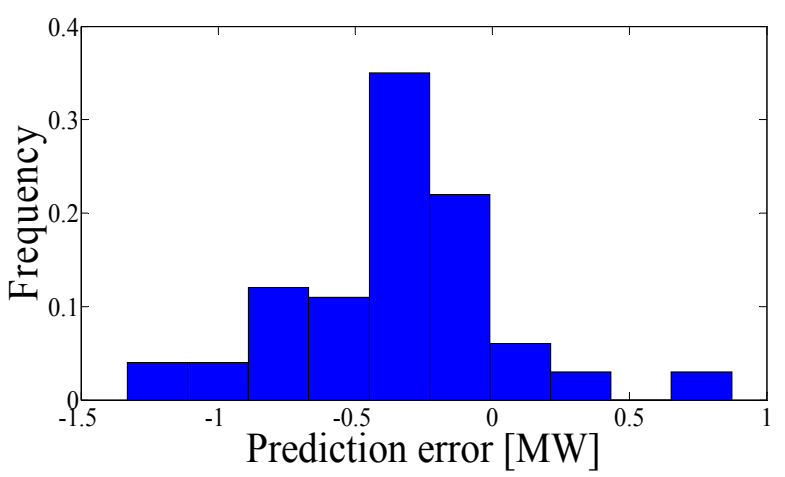

(b1)

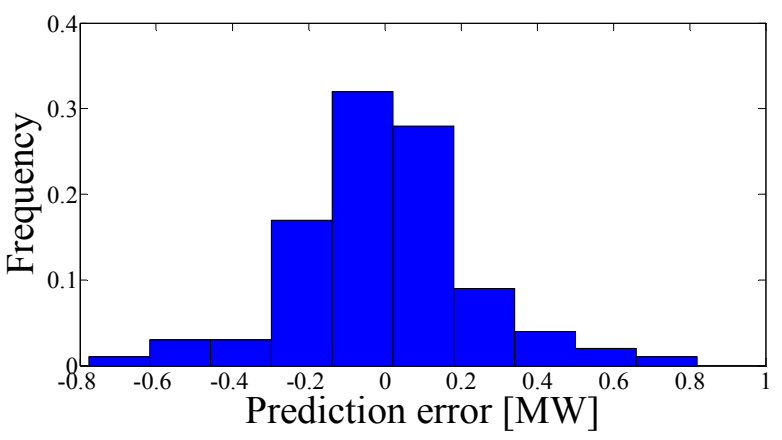

(b2)

Figure 4. Error of wind farm in Spain. (a) Prediction errors. (b1)Error distribution before correction. (b2) Error distribution after correction.

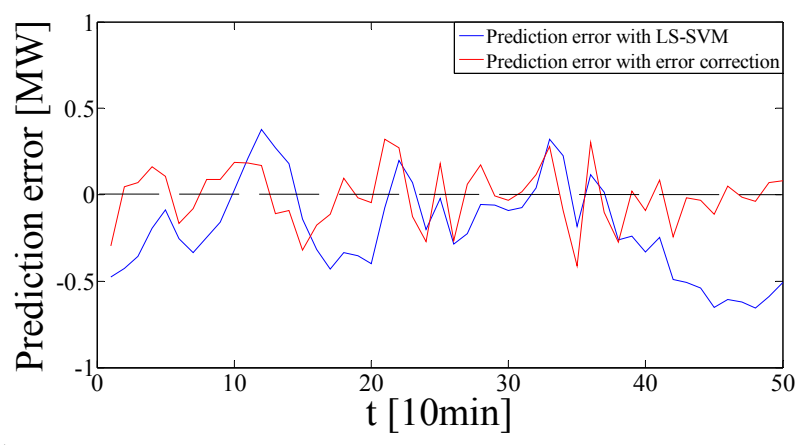

(a)

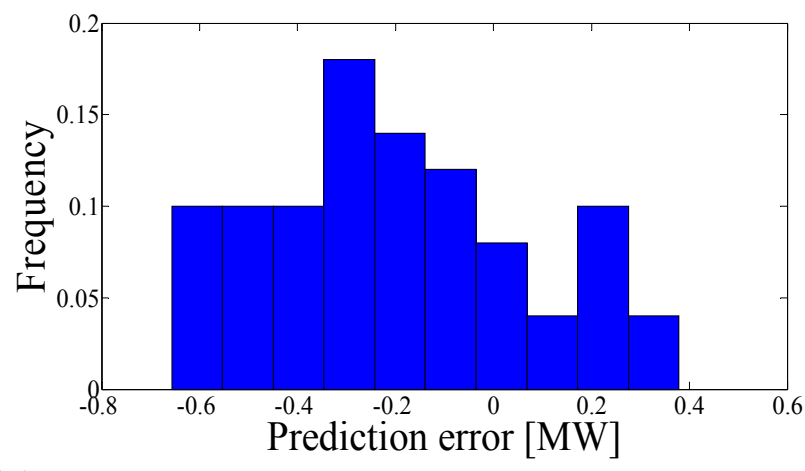

(b1)

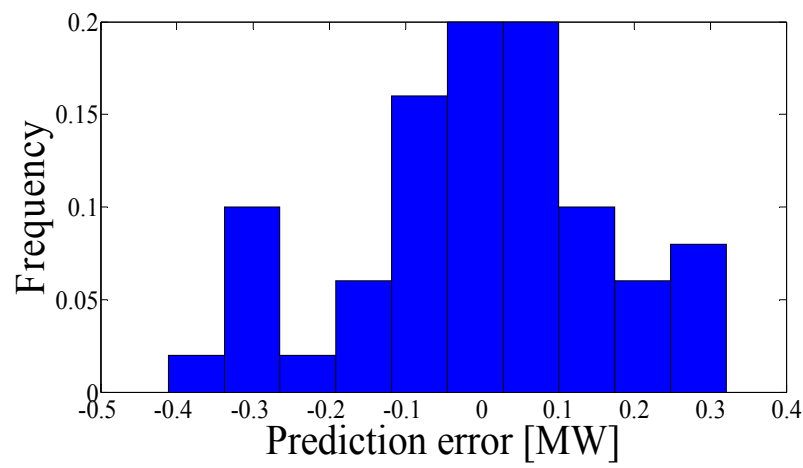

(b2)

Figure 5. Error of wind farm in China. (a) Prediction errors. (b1)Error distribution before correction. (b2) Error distribution after correction.

The good power prediction performance of the LS-SVM model after error correction can be seen directly from the results of the prediction curve and the error distribution of Figure 3-5. At the same time, the prediction accuracy of the four models can be clearly seen from the quantitative results of Table II. The biggest change of errors between the LSSVM model and LS-SVM with error correction occurred in the wind farm of Spain, MAE and MSE respectively reached 0.1692 and 0.0532 . Compared with the simple LSSVM model, the mean absolute error of the LS-SVM prediction model with error correction was decreased by $59.7 \%$ and $52.0 \%$ respectively and the mean square error and mean absolute percentage error were reduced by at least $74 \%$ and $68.4 \%$ respectively. Through the above analysis, it can be seen whether using Spanish wind power data or Chinese wind power data, the LS-SVM model with error correction was better than the simple LS-SVM model. This once again confirmed the good performance and broad practicability of the LS-SVM prediction model with error correction. In short, the least squares support vector machine based on error correction proposed in this paper is superior to the traditional prediction models (RBF, Elman, LSSVM), which has a wide range of popularity.

TABLE II . ERROR INDEX OF PREDICTION MODELS

\begin{tabular}{|c|c|c|c|c|c|c|c|c|}
\hline \multirow{2}{*}{ Model } & \multicolumn{3}{|c|}{ wind farm in Spain } & & \multicolumn{3}{c|}{ wind farm in China } \\
\cline { 2 - 9 } & EBror index & Elman & LS-SVM & $\begin{array}{c}\text { LS-SVM with } \\
\text { error } \\
\text { correction }\end{array}$ & RBF & Elman & $\begin{array}{c}\text { LS-SVM } \\
\text { error } \\
\text { correction }\end{array}$ \\
\hline$M A E(M W)$ & 0.3900 & 0.5363 & 0.4203 & 0.1692 & 0.3943 & 0.3135 & 0.2818 & 0.1354 \\
\hline$M S E\left(M^{2}\right)$ & 0.2807 & 0.3509 & 0.2652 & 0.0532 & 0.2017 & 0.1346 & 0.1117 & 0.0282 \\
\hline$M A P E(\%)$ & 23.18 & 26.33 & 33.59 & 10.60 & 69.85 & 36.38 & 67.86 & 16.98 \\
\hline
\end{tabular}




\section{CONCLUSION}

Energy problem is a top priority for all countries. Such as renewable energy assistance program in Norway and Sweden, energy transformation strategy of Germany, development strategy of Denmark aiming to get rid of the fossil energy in 2050, the core idea of which is the reconstruction of power system in the future, that is to construct the future power system fully using renewable power. Wind energy is bound to become the first choice for the new energy sources in the future with its mature technology, rich source and characteristics of environmentfriendly. However, wind power is still facing a number of challenges, including wind turbine load control [24] and its unstable power output. The difficult storage and instability of wind energy will affect wind power output and the safe operation of the wind farm, making it more important to further improve the accuracy of wind power prediction. The formation of wind is nonlinear in nature, and subtle changes will interfere with the formation of the atmospheric system. Because the traditional power prediction method cannot met the prediction accuracy, and it had been found that the error of adjacent time is similar to the error level, so the error correction was introduced into the LS-SVM model in this paper to establish the least square support vector machine power prediction model based on error correction and the power data of two wind farms in Spain and China were used for simulation. The results showed that the error correction model is better than the traditional prediction models (RBF, Elman, LS-SVM) and significantly improved the overall level of wind power prediction, having a high practical value.

In view of the significant effect of error correction, we will continue to explore the error correction model for other numerical prediction methods of wind power in the next step. At the same time, we will also fully consider the temperature, pressure, humidity and other climatic conditions of wind farm and try to put the statistical characteristics of wind power series and the dynamic properties of atmospheric turbulence into the wind prediction model to improve the original forecast.

\section{ACKNOWLEDGMENT}

The authors thank Distinguished Prof. Eugen COCA and the anonymous referees for the thoughtful and constructive suggestions that led to a considerable improvement of the paper.

\section{REFERENCES}

[1] A. Y. Sultan, C. Yassine, A. B. Abdullah, G. Adel, "Nested ensemble NWP approach for wind energy assessment," Renewable Energy, Vol. 37, pp. 150-160, Jan. 2012. doi:10.1016/j.renene.2011.06.014

[2] V. Prema, K. U. Rao, "Development of statistical time series models for solar power prediction," Renewable Energy, Vol. 83, pp. 100-109, Nov. 2015. doi:10.1016/j.renene.2015.03.038

[3] H. R. Zhao, S. Guo, "An optimized gray modal for annual power load forecasting," Energy, Vol. 107, pp. 272-286, Jul. 2016. doi:10.1016/j. energy.2016.04.009

[4] C. D. Zuluaga, M. A. Álvarez, E. Giraldo, "Short-term wind speed prediction based on robust Kalman filtering: An experimental comparison," Applied Energy, Vol. 156, pp. 321-330, Oct. 2015. doi:10.1016/j.apenergy.2015.07.043

[5] H. P. Liu, E. Erdem, J. Shi, "Comprehensive evaluation of ARMAGARCH (-M) approaches for modeling the mean and volatility of wind speed," Applied Energy, Vol. 88, pp. 724-732, Mar. 2011. doi:10.1016/j.apenergy.2010.09.028

[6] C. Fan, S. Liu, "Wind Speed Forecasting Method: Gray Related Weighted Combination with Revised Parameter," Energy Procedia, Vol. 5, pp. 550-554, Apr. 2011. doi:10.1016/j.egypro.2011.03.096

[7] P. Ramasamy, S. S. Chandel, A. K. Yadav, "Wind speed prediction in the mountainous region of India using an artificial neural network model," Renewable Energy, Vol. 80, pp. 338-347, Aug. 2015. doi:10.1016/j.renene.2015.02.034

[8] H. Chitsaz, N. Amjady, H. Zareipour, "Wind power forecast using wavelet neural network trained by improved Clonal selection algorithm," Energy Conversion and Management, Vol. 89, pp. 588598, Jan. 2015. doi:10.1016/j.enconman.2014.10.001

[9] M. A. Mohandes, T. O. Halawani, S. Rehman, A. A. Hussain "Support vector machines for wind speed prediction," Renewable Energy, Vol. 29, no. 6, pp. 939-947, May. 2004 doi:10.1016/j.renene.2003.11.009

[10] K. G. Sheela, S. N. Deepa, "Neural network based hybrid computing model for wind speed prediction," Neurocomputing, Vol. 122, pp 425-429, Dec. 2013. doi:10.1016/j.neucom.2013.06.008

[11] K. Chen, J. Yu, "Short-term wind speed prediction using an unscented Kalman filter based state-space support vector regression approach," Applied Energy, Vol. 113, pp. 690-705, Jan. 2014. doi:10.1016/j. apenergy.2013.08.025

[12] Y. G. Zhang, J. Y. Yang, K. C. Wang, Z. P. Wang, Y. D. Wang, "Improved wind prediction based on the Lorenz system." Renewable Energy, Vol. 81, pp. 219-226, Sep. 2015. doi:10.1016/j.renene.2015. 03.039

[13] Y. G. Zhang, J. Y. Yang, K. C. Wang, Y. D. Wang. "Lorenz Wind Disturbance Model Based on Grey Generated Components." Energies, Vol. 7, no. 11, pp. 7178-7193, Nov. 2014.doi:10.3390/en7117178

[14] Y. G. Zhang, J. Y. Yang, K. C. Wang, Z. P. Wang, "Wind Power Prediction Considering Nonlinear Atmospheric Disturbances." Energies, Vol. 8, pp. 475-489, Jan. 2015.doi: 10.3390/en8010475

[15] Z.Y. Su, J.Z. Wang, H.Y. Lu, G. Zhao, "A new hybrid model optimized by an intelligent optimization algorithm for wind speed forecasting," Energy Conversion and Management, Vol. 85, pp. 443 452, Sep. 2014. doi:10.1016/j.enconman.2014.05.058

[16] M. Monfared, H. Rastegar, H. M. Kojabadi, "A new strategy for wind speed forecasting using artificial intelligent methods," Renewable Energy, Vol. 34, no.3, pp. 845-848, Mar. 2008. doi:10.1016/j.renene. 2008.04.017

[17] C. Ren, N. An, J. Z. Wang, et al., "Optimal parameters selection for BP neural network based on particle swarm optimization: A case study of wind speed forecasting," Knowledge-Based Systems, Vol. 56, pp. 226-239, Jan. 2014. doi:10.1016/j.knosys.2013.11.015

[18] S. B. Ghosn, F. Drouby, H. M. Harmanani, "A Parallel Genetic Algorithm for the Open-Shop Scheduling Problem Using Deterministic and Random Moves," International Journal of Artificial Intelligence, Vol. 14, no. 1, pp. 130-144, 2016.

[19] A. Mellit, A. M. Pavan, M. Benghanem, "Least squares support vector machine for short-term prediction of meteorological time series," Theor Appl Climatol, Vol. 111, pp. 297-307, May. 2013 doi:10.1007/s00704-012-0661-7

[20] X. H. Yuan, C. Chen, Y. B. Yuan, Y. H. Huang, "Short-term wind power prediction based on LSSVM-GSA modal," Energy Conversion and Management, Vol. 101, pp. 393-401, Sep. 2015. doi:10.1016/j. enconman.2015.05.065

[21] M. A. Ramírez-Ortegón, V. Märgner, E. Cuevas, R. Rojas, "An optimization for binarization methods by removing binary artifacts," Pattern Recognition Letters, Vol. 34, pp. 1299-1306,Aug. 2013. doi:org/10.1016/j.patrec.2013.04.007

[22] R. E. Precup, S. Preitl, "Optimization criteria in development of fuzzy controllers with dynamics," Engineering Applications of Artificia Intelligence, Vol. 17, pp. 661-674, Aug. 2004 doi:10.1016/j.engappai.2004.08.004

[23] Z. Li, L. Ye, Y. N. Zhao, X. R. Song, et al., "Short-term wind power prediction based on extreme learning machine with error correction,' Protection and Control of Modern Power Systems, Vol. 1, pp. 2-8, Jun. 2016. doi:10.1186/s41601-016-0016-y

[24] E. A. Bossanyi, "Wind Turbine Control for Load Reduction," Wind Energ, Vol. 6, pp. 229-244, Jun. 2003. doi:10.1002/we.95 